\title{
Prevention of depression and sleep disturbances in elderly with memory-problems by activation of the biological clock with light - a randomized clinical trial
}

\author{
Els IS Most ${ }^{1,2^{*}}$, Philip Scheltens ${ }^{2}$, Eus JW Van Someren ${ }^{1,3,4}$
}

\begin{abstract}
Background: Depression frequently occurs in the elderly and in patients suffering from dementia. Its cause is largely unknown, but several studies point to a possible contribution of circadian rhythm disturbances. Post-mortem studies on aging, dementia and depression show impaired functioning of the suprachiasmatic nucleus (SCN) which is thought to be involved in the increased prevalence of day-night rhythm perturbations in these conditions. Bright light enhances neuronal activity in the SCN. Bright light therapy has beneficial effects on rhythms and mood in institutionalized moderate to advanced demented elderly. In spite of the fact that this is a potentially safe and inexpensive treatment option, no previous clinical trial evaluated the use of long-term daily light therapy to prevent worsening of sleep-wake rhythms and depressive symptoms in early to moderately demented home-dwelling elderly.

Methods/Design: This study investigates whether long-term daily bright light prevents worsening of sleep-wake rhythms and depressive symptoms in elderly people with memory complaints. Patients with early Alzheimer's Disease (AD), Mild Cognitive Impairment (MCI) and Subjective Memory Complaints (SMC), between the ages of 50 and 75, are included in a randomized double-blind placebo-controlled trial. For the duration of two years, patients are exposed to $\sim 10,000$ lux in the active condition or $\sim 300$ lux in the placebo condition, daily, for two half-hour sessions at fixed times in the morning and evening. Neuropsychological, behavioral, physiological and endocrine measures are assessed at baseline and follow-up every five to six months.
\end{abstract}

Discussion: If bright light therapy attenuates the worsening of sleep-wake rhythms and depressive symptoms, it will provide a measure that is easy to implement in the homes of elderly people with memory complaints, to complement treatments with cholinesterase inhibitors, sleep medication or anti-depressants or as a stand-alone treatment.

Trial registration: ISRCTN29863753

\section{Background}

As the western population is increasingly aging, problems connected with old age will dominate health care. Depression, one of the most prevalent psychiatric disorders, is expected to take an even more prominent position than presently, as the risk for developing depression increases with old age. Depressive symptoms are present in almost one third of the elderly population

\footnotetext{
* Correspondence: e.most@vumc.nl
${ }^{1}$ Netherlands Institute for Neuroscience, an Institute of the Royal Netherlands

* Correspondence: e.most@vumc.nl
${ }^{1}$ Netherlands Institute for Neuroscience, an Institute of the Royal Netherlands Academy of Arts and Sciences, Meibergdreef 47, 1105 BA Amsterdam, the Netherlands
}

(c) 2010 Most et al; licensee BioMed Central Ltd. This is an Open Access article distributed under the terms of the Creative Commons Attribution License (http://creativecommons.org/licenses/by/2.0), which permits unrestricted use, distribution, and reproduction in any medium, provided the original work is properly cited.

and major depression may be present in up to $4 \%[1,2]$. Furthermore, once present, the prognosis for elderly with depression is poor [3].

In 2007, an estimated 27 million people worldwide were diagnosed with Alzheimer's Disease (AD), the most common form of dementia, and this number is expected to quadruple by 2050 [4]. Although the most prominent symptom is cognitive decline, in time other distinctive symptoms develop, such as sleep-wake rhythm disturbances [5-10], behavioral disturbances including agitation and psychosis [11-15] and mood disturbances, notably depression $[16,17]$. 


\section{Depression in dementia}

Depression prevalence can reach up to $18 \%$ in $\mathrm{AD}$ patients $[18,19]$. The occurrence of a major depressive episode in $\mathrm{AD}$ is as high as $20-25 \%$, and minor depressive symptoms occur in an additional 20-30\% [20,21]. Once they are present, depressive symptoms are persistent $[3,22]$. This shows that depression is common, especially in early/mild and moderate AD patients. Although Verkaik (2007) stated that the prevalence of depression between mild and severe dementia do not differ much [23], this is difficult to assess in severe dementia, something which may indicate an underestimation of the actual occurrences.

The consequences of depression can be considerable. Depression not only has a significant impact on the patient's quality of life and activities of daily life $[16,17,24]$, it is also one of the major causes for depression in caregivers $[25,26]$, all of the above contributing to early institutionalization of the patient. Additionally, the presence of mood disturbances is associated with increased mortality [27].

The etiology of depression in AD is still largely unknown. Being aware of having AD or the loss of (cognitive) function seems unrelated [28]. There is some evidence that the neuropathological features of AD play a role; Alzheimer pathology in the locus coeruleus [29] may be involved, as well as the dorsal raphe serotonergic nuclei [30]. However, newer studies have failed to replicate these data [31,32], and the precise part of AD neuropathology in the risk of developing depression is still an ongoing discussion.

A family history of depressive symptoms is the most prominent risk factor for developing depression in AD. A personal history of depression, gender and a young onset of AD also contribute significantly [33]. A key predictor of depressive symptoms is the presence of sleep disturbances [34-37]. Elderly experience more difficulties with sleeping than the young [38], increasing the probability of developing mood problems. As sleep problems become very distinctive in $A D$ [5], it can be assumed that the chances of developing mood disorders are even higher.

\section{How light therapy might work}

Light activates retinal ganglion cells which in turn excite the hypothalamic suprachiasmatic nucleus (SCN), the biological clock of the brain, via the retinohypothalamic tract. The SCN is the circadian pacemaker, generating and synchronizing biochemical and behavioural rhythms, for example, the regulation of melatonin via the paraventricular nucleus and cortisol through the hypothalamic-pituitary-adrenal axis [39]. Maintaining an endogenous rhythm of approximately 24.2 hours, the $\mathrm{SCN}$ is synchronized to the environmental day and night by light, to which it is extremely sensitive. A regular input is therefore necessary to ensure optimal coupling to the environmental 24-hour light-dark cycle. During the aging process, ocular light transmission may be impaired by age-related deficiencies in the eye (e.g., cataract) and degeneration of the optic nerve, creating a demand for higher light intensities in order to maintain a sufficient input to the SCN [8]. Without this increase, the SCN input gradually diminishes, which may be involved in the finding that $\mathrm{SCN}$ neurons show decreased activation at high age, and even more so in demented elderly [40]. These changes are likely to affect the pacemaker function of the $\mathrm{SCN}$, showing up as arrhythmia in the sleep-wake cycle. Indeed, the severity of sleep-wake rhythm disturbances is strongly correlated with the loss of vasopressin neurons in the SCN [41].

Combined with the neuropathological effects of $\mathrm{AD}$, decreasing SCN responsiveness even further due to increased cell(pathway) loss [42], previous studies suggest that it is possible to reactivate the $\mathrm{SCN}$ and improve sleep-wake rhythms by applying extra light, as will be summarized briefly in the next paragraph.

\section{Bright light therapy in AD}

Since the early 1990s several researchers have evaluated the effect of bright light in demented elderly. Frequently reported positive effects are improvements of disturbed sleep and activity rhythms [12,43-48]. However, some studies fail to show these effects [49-51].

Only a handful of researchers have studied the effects of light therapy on mood in AD. Whereas efficacy could not be demonstrated in short-term studies with either three weeks of dusk and dawn simulation [52] or four weeks of one hour of morning light [12], Dowling et al did find small positive effects after ten weeks of one hour of morning light [53]. Even more pronounced effects on mood were found with long-term (up to 3,5 years) of whole-day bright light [43]. Although these findings concertedly suggest that effects may take weeks to develop, the best methodological approach to light therapy in $\mathrm{AD}$ is still unknown. Also, only a limited number of the studies on light therapy in dementia classify as randomized clinical trials. Furthermore, of all light therapy studies, the study of Riemersma et al (2008) has been the only one investigating long-term effects (up to 3,5 years). Finally, only a few studies investigated effects in community-dwelling patients [49]. Thus, additional randomized clinical trials are warranted, especially for investigating effects of longterm treatment on mood in community-dwelling elderly with memory complaints, either diagnosed with early Alzheimer's disease or with conditions that may develop into Alzheimer's disease.

\section{Advantages of light therapy}

Light therapy provides a safe treatment option, with only mild side effects [54]. One study even reported a 
decrease in the frequency of common health complaints with bright light [43]. In non-demented people, light therapy is an accepted and validated treatment for seasonal affective disorder, but has also been reported to be effective in non-seasonal depression [55]. Acceptance among patients is high and it is a feasible option for patients who do not tolerate or are opposed to medication, or when medication is not effective [56].

\section{Ocular safety}

The light intensity used is well within the range of normal daylight exposure. Furthermore, the filters applied on the light devices reduce the ocular UV and blue light hazard to far below the risks of exposure to natural daylight. Although not specifically examined in dementia patients, its long- term safety has been demonstrated in seasonal affective disorder patients by ophthalmologic examinations after exposures of up to 1250 hours [57].

Given these considerations, the aims of the current study are to investigate whether long-term daily bright light exposure is an effective treatment in home-dwelling elderly with memory problems who are at an increased risk for the development or worsening disturbed mood and sleep.

\section{Methods and design}

\section{General objectives}

The purpose of this study is to investigate the following primary hypothesis:
1. Long-term daily bright light exposure attenuates the occurrence of depressive symptoms.

Secondary hypotheses are:

1. Long-term daily bright light exposure attenuates the occurrence of sleep-wake rhythm disturbances.

2. Long-term daily bright light exposure ameliorates cognitive deficits.

3. Long-term daily bright light exposure ameliorates caregiver burden.

4. The effects of light on mood and cognition are in part mediated by its effect on the circadian pacemaker, as read out from the 24-hour rhythms in activity, body temperature, heart rate and cortisol.

\section{Procedure}

After a baseline measurement $\left(\mathrm{T}_{0}\right)$, all patients receive either a placebo or an active light box (see interventions and randomization). They are then followed-up for a maximum of four half yearly visits $\left(T_{1}, T_{2}, T_{3}\right.$ and $\left.T_{4}\right)$ (figure 1, Design and protocol). For comparison, a baseline assessment is obtained in healthy controls at $\mathrm{T}_{0}$ only.

In short, at all assessments $\left(\mathrm{T}_{0}\right.$ to $\left.\mathrm{T}_{4}\right)$ patients visit the VU University medical center for two hours, which start with an explanation of the procedures and administration of the questionnaires (figure 1, Protocol).

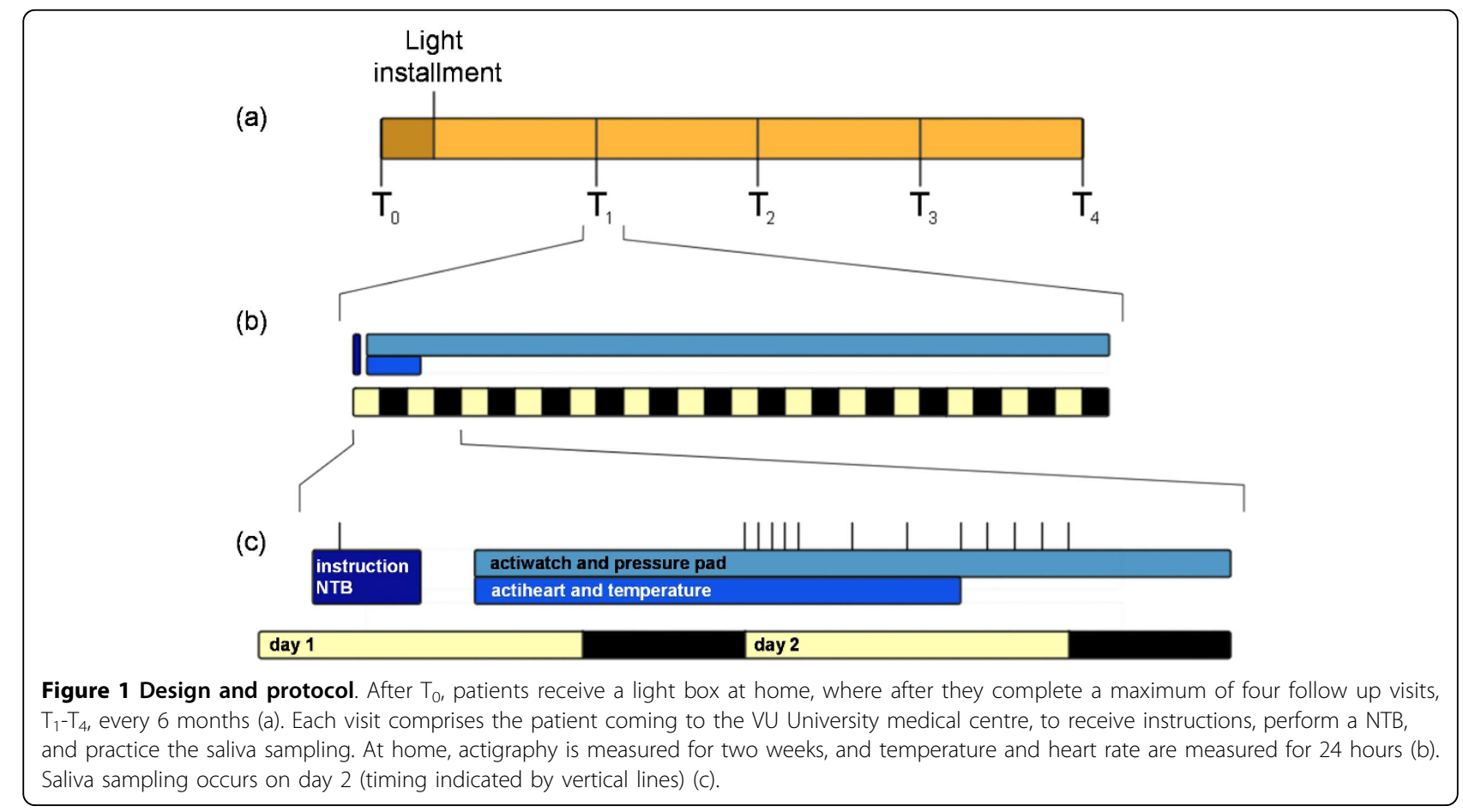


Depressive symptoms are assessed with the Geriatric Depression Scale. To measure sleep, patients complete the Athens Insomnia Scale, the Dutch Sleep Disorders Questionnaire and the Pittsburg Sleep Quality Index. A neuropsychological test battery is administered as well. The primary caregiver fills out the Zarit Burden Interview and the Self-Perceived Pressure from Informal Care.

Subsequently, in order to measure skin temperature, 9 temperature loggers are placed on the body (thighs, abdomen, soles of the hands and feet), to be worn for 24 hours. During this time patients also wear a heart rate recorder. An actiwatch is placed on the non-dominant wrist for two weeks to monitor activity rhythms and to estimate sleep. In the same period, a pressure pad connected to a data logger is placed on the patients' bed at shoulder-level, underneath their bed-sheet, to determine the period people spend in bed. The logger, which is placed beside the bed, includes a light sensor for a determination of lights out time. On the second day of the protocol, patients collect 11 saliva samples, from which the diurnal pattern of cortisol levels is determined.

All proceedings are discussed in detail below.

\section{Participants}

Patients are recruited (1) by screening patients diagnosed or treated at the Alzheimer Center of the VU University medical center, Amsterdam, (2) referred from a number of collaborating neurologists and gerontologists in the North Holland region, (3) through reactions to an advertisement in a popular magazine for elderly (PLUS), (4) through enlisting at the "Ontmoetingcentra for Dementerenden" (Meeting Centres Support Program) in North Holland. Patients' partners and volunteers from the local community are included in the control group.

\section{Inclusion and exclusion criteria}

All patients must be between 50 and 80 years of age. Because we aim to study the effects in the earliest stage of developing memory problems we include not only people with a clinical diagnosis of probable (presenile) Alzheimer's Disease ((pre-)AD) but also people with Mild Cognitive Impairment (MCI), diagnosed by a neurologist or gerontologist according to the DSM-IV [58] or the NINCDS-ADRDA criteria [59] and the MCI- standard set by Petersen et al (2001) [60] as well as visitors of the Alzheimer center who do not fulfill the above mentioned criteria but suffer from Subjective Memory Complaints (SMC). A Mini Mental State Exam (MMSE) score of or above 14 is required for all participants at inclusion [61]. No restrictions are made for medication use.

Patients are excluded from participation in the study if diagnosed with (1) any other neurological disorder, including narcolepsy, (2) any psychiatric disorder, with the exception of mild depressive symptoms, (3) serious problems with activities of daily living (ADL), (4) sleep apnoea or restless legs syndrome or (5) a serious eye disease incompatible with light therapy, such as aphakia or retinitis pigmentosa. Healthy controls must be free of any clinical diagnosis, and not have subjective memory complaints. They also must meet the exclusion criteria mentioned above. They are selected to match the age and gender distribution of the patients. The MMSE cutoff score is $\geq 28$.

\section{Interventions}

Light boxes are installed at the patients' home, in a room of their preference. In the active condition, light boxes provide a light intensity of $\sim 10,000$ lux measured at the eye level in the gaze direction. A valid placebo condition was created by random assignment of half of the participants to a seemingly identical light box designed to produce light levels not exceeding \pm 300 lux at eye level in the gaze direction. In these light boxes, the reflecting mirrors were not placed in an optimal position, full-band filters were applied and tubes of lower intensity were used. Intensities are quantified using a lux meter.

During the two-year treatment period, participants expose themselves daily to the light in sessions lasting 30 minutes every morning and evening. During this time patients do activities of their own choice such as reading, eating, watching television or working at a computer. To limit day-to-day variation in exposure timing, yet providing some individual flexibility, patients decide on a convenient fixed time-window for both the morning and evening sessions. They are free to choose their 30 minute exposures during these 90 minutes time-windows, when light is automatically switched on and cannot be switched off. If desired, lights can be switched on and off or dimmed manually at all other times of the day for use as normal lighting. The timer automatically adjusts to daylight-saving time.

\section{Compliance}

Light therapy compliance is measured by a motion detector connected to the light box. A registration logger (DALI logger GOAL 10) is connected to a motion detector (DALI multisensor DCMC302), located on the light box for two weeks at assessment $\mathrm{T}_{1}$ to $\mathrm{T}_{4}$. Also, patients and their partners are asked at every assessment whether long periods of absence (e.g. holidays) or other compliance issues (e.g. patient being unable to remain seated for 30 minutes or illness) have occurred.

\section{Sample size}

Under the assumption of four successful follow-up assessments and a within-subject correlation of $\mathrm{r}=0.40$, 
72 participants in total would yield, at a two-sided alpha $<0.05$, a power of 0.81 to detect a medium effect size of $\mathrm{d}=0.35$ for main effects [62], i.e. a difference of 0.35 of the pooled standard deviation.

\section{Randomization}

Randomization and installation of the lights is performed by a technician not involved in the study (M. Oomstee, Netherlands Institute for Neuroscience, Amsterdam) and kept concealed. Random assignments are generated using Excel spreadsheet software (Microsoft Corporation, Redmont, USA) Codes are revealed to the researchers only after completion of the study and subsequent data reduction and processing steps.

\section{Statistical analysis}

Prior to analyses, an automated procedure implemented in the Excel spreadsheet software (Microsoft Corporation, Redmont, USA) will be used to screen for, and exclude, extreme outliers in the outcome measure value. Excluded are observations that are at least three interquartile ranges lower than the first quartile or higher than the third quartile. Second, within-subject extremely outlying temporary changes that last only one observation are excluded. To do so, for all changes, the minimal difference to the previous or next observed value is calculated, and the distribution of these minimal differences is evaluated. Excluded are observations for which this minimal difference is three interquartile ranges lower than the first quartile or higher than the third quartile.

Mixed effect regression analysis [62] is the analysis of choice for realistic long-term data sets in psychiatry and elderly chronic care populations with variable number of available observations due to dropout or missing observations $[63,64]$. The analyses are performed with the MLwiN software (version 2.0, Institute of Education, London, UK) and account for the two-level nested structure of the dataset, i.e. a variable number of observations of five or less nested within participants. Light is dummy coded indicating the assignment to active treatment. For the primary outcome measure and other variables without expected gradually increasing response over time, a second dummy codes whether the observation is prior to or during treatment. Analyses apply the full factorial design with the factors light assignment group, post vs. pre assessment, and their interaction. The latter effect is the effect of interest. For secondary outcome measures with an expected gradually increasing response change over time, both pre-post treatmenteffects (i.e. independent of time) and time-by-treatment effects (i.e., treatment effects evolving slowly over time) are evaluated. In ancillary analyses, the regression models allowed for evaluation of linear changes over time, and for modification of level, time course and treatment effect by missing data patterns. Logistic mixed effect regression is applied in order to evaluate possible prepost group differences and group by time interactions in binary outcomes such as the prescription of medication.

\section{Approval}

This study follows the Helsinki Declaration's principles, meaning that all patients sign a written informed consent stating that participation is voluntary and that participation can be withdrawn at any time, without any negative consequences concerning their current or future medical treatment. Approval has been obtained from the medical ethical committee of the VU University medical center (Protocol 2005/10).

\section{Primary Outcome measure}

Depressive symptom severity during $T_{1}$ to $T_{4}$ relative to $\mathrm{T}_{0}$ as assessed using the 30 -item version of the Geriatric Depression Scale (GDS).

\section{Secondary Outcome Measures}

Secondary outcome measures are (1) sleep efficiency and sleep-wake rhythm amplitude and variability as estimated from actigraphy; (2) cognitive performance as assessed by the NTB (3) caregiver burden as assessed using the questionnaires Zarit Burden Interview and Self-Perceived Pressure from Informal Care. All contrasts compare $\mathrm{T}_{1}$ to $\mathrm{T}_{4}$ relative to $\mathrm{T}_{0}$. In order to obtain insight in underlying mechanisms, the study will evaluate whether the effects of light on mood and cognition are in part mediated by its effect on the circadian pacemaker, as read out from the rhythms in activity, body temperature and cortisol.

\section{Adverse effects}

At each assessment patients are asked to report any side effects. Also, between three to six weeks after the light box installment, patients are contacted by telephone to ask them about any acute negative effects.

After finishing the study, or when stopping prematurely, all patients and partners received an evaluation form including a screening for adverse effects (shaking hands, stomach cramps, sweating, hunger, weakness, drowsiness, constipation, dizziness, 'sleeping' limps, nausea, irritability, hyperactivity, inability to sleep, headache and irritation to the eyes) rated by a visual analog scale.

\section{Cortisol measures}

The saliva cortisol profile during wake is obtained at all assessments. The sampling protocol captures the morning cortisol awakening response by four saliva samples at wake up time, plus 20 minutes, plus 40 minutes and plus 60 minutes. If patients wake up before 07:00, an 
additional collection is made at 08:00. The pre-sleep levels are assessed at bedtime, minus 1 hour, minus 2 hours, minus 3 hours and minus 4 hours. Additional samples are collected at 12:00, 16:00 and, if not already sampled for the pre-sleep levels, at 20:00. Saliva is sampled using Salivettes (Sarstedt AG \& Co, Nümbrecht, Germany), which are stored in the patient's freezer until sent back to the investigator. Transportation via mail is a convenient way of returning material, as patients live all over the Netherlands and cortisol levels are not influenced significantly by some days outside the freezer [65,66]. Patients are instructed to rinse their mouth with water and to remain seated while collecting. No eating or drinking is allowed within twenty minutes adjacent to sampling and patients are advised to refrain from eating chocolate, bananas and eggs and from drinking caffeinated coffee or black tea on the day of sampling. No restriction is placed on smoking.

At the lab, samples are stored at $-20^{\circ} \mathrm{C}$ until all samples can be analyzed simultaneously [67], using the Spectria Cortisol RIA (Orion Diagnistica Oy, Espoo, Finland).

\section{Actigraphy and bedtimes}

Sleep and activity rhythms are quantified using the Actiwatch (CamNtech Ltd., Cambridge, UK) [68], which patients wear on their non-dominant wrist for two weeks [69]. During this time, to determine bedtimes, patients sleep on a pressure sensitive pad consisting of two conductive layers separated by a perforated thin layer of insulating foam, all covered in vinyl (317-140, RS-components, Haarlem, The Netherlands) [70]. When pressure is applied to the pad, the two conductive layers make contact through the pores of the insulating layer, providing a switch. The pressure pad is connected to a Hobo U12-06 logger with a Light Dependent Resistor (LDR, NSL-4962, Silonex, Montreal, Canada) fixed on it. Patients also keep a sleep log, registering bedtimes, lights-out times, whether partners keep the light on, wake up and get up times and any daytime naps.

Sleep parameters are obtained using the Actiware, version 5.57.0006 (Respironics, Inc, Murrysville, USA). Bedtimes extracted from the pressure pad data are implemented in the analysis and the software automatically calculates sleep efficiency (SE), total sleep duration (TST), nocturnal restlessness, average durations of nocturnal awakenings, and of uninterrupted sleep.

Several aspects of the diurnal activity rhythms are calculated as previously described [71,72]: the constancy of the 24-hour sleep-wake pattern over days called interdaily stability (IS); the rhythm fragmentation called intradaily variability (IV); the hourly average of minutes with activity during the 5 minimally active hours (L5); the hourly average of minutes with activity during the 10 most active hours (M10); and the absolute amplitude measure (AMP) of the rhythm calculated as the difference between M10 and L5. The relative amplitude (RA) is calculated by dividing AMP by the sum of L 5 and M10.

\section{Temperature}

Skin Temperature (Ts) is assessed using iButtons (type DS1921H, Maxim Integrated Products, Inc., Sunnyvale, USA), located at 9 positions (hands, feet, clavicles, abdomen and thighs) for 24 hours, with a recording interval of one minute [73]. From these 9 positions $\mathrm{Ts}_{\text {proximal }}$ (abdomen, clavicles and thighs) and $\mathrm{Ts}_{\text {distal }}$ (hands and feet) are calculated [74]. Averages are calculated over the out-of-bed and in-bed period, determined with the pressure pad data described above. Patients also wear a heart rate (HR) recorder (Actiheart, CamNtech Ltd., Cambridge, UK) for $24 \mathrm{hr}$. HR variability can give an approximation of heat production from heart rate as well as a possibility to investigate the sympathetic to parasympathetic balance.

In addition, the environmental temperature of the bedroom and living room is measured with iButtons (type DS1922L, Maxim Integrated Products, Inc., Sunnyvale, USA) for 14 days with a 10 minute interval at one assessment $\left(T_{2}\right.$ or $\left.T_{4}\right)$. Outdoor environmental temperatures during all assessments are downloaded from the Royal Dutch Meteorological Institute website http:// www.knmi.nl.

\section{Neuropsychological tests}

At all assessments $T_{0}$ to $T_{4}$, patients complete the Mini Mental State Examination (MMSE) [61] and a neuropsychological test battery (NTB), aimed at covering a wide range of cognitive domains: short and long term verbal memory (the 15 Word List, [75]; the MIS+, developed in-house at the VUmc Alzheimer Center, no published norm values); semantic memory (WAIS Information from the Wechsler Adult Intelligence Scale (WAIS III) [76]); working memory (Number Sequences, [76]); visual memory (the Visual Association Test (VAT) [77]). Furthermore, patients are tested for interference (The Stroop Color/word Test [78], verbal fluency (Category Fluency [79]), attention and planning (The Trailmaking Test $A$ and $B$ [80]).

Additionally, at $\mathrm{T}_{0}$ visual memory is more extensively tested (Rey Complex Figure [81]; WAIS III subtest Symbol Substitution [76]); as well as motor perseveration (Amsterdam Dementia Screening subtest Meander [82]); planning (WAIS Revised subtest Maze (simplified) [83]) and flexibility and working memory (the Rule Shift subtest from the Behavior Assessment for Dysexecutive Symptoms (BADS) [84]). 


\section{Questionnaires}

All questionnaires are presented in Dutch and completed at home.

The primary outcome of depression is measured with the Geriatric Depression Scale (GDS), using the complete 30 items version [85]. The GDS is a list of statements and patients are asked to rate whether these statements are applicable to them during the last week, answering 'yes' or 'no'. The range of the cumulative score is 0 to 30 and scores are labeled as follows: 0-9 as "not depressed", 10-19 as "mildly depressed", and 20-30 as "severely depressed".

The Athens Insomnia Scale (AIS) is a questionnaire consisting of eight items, based on the criteria of the 10th edition of the International Classification of Diseases, (ICD-10) [86]. The total score ranges from 0 (absence of any sleep-related problem) to 24 (severe degree of insomnia). The responders are requested to rate an item as positive (i.e. to choose among rating options 1, 2 and 3) only if they have experienced any sleep difficulties at least three times a week during the last month. This frequency is consistent with the ICD-10 criteria for insomnia. The AIS has a cut off score of $\geq 5$, the higher scores being suggestive of a diagnosis of insomnia.

The Pittsburg Sleep Quality Index (PSQI) is a 19-item scale assessing overall sleep quality over a 1-month period, with scores ranging from 0 to 21 (higher scores indicating worse sleep quality) [87]. It scores subjective sleep quality, sleep latency, sleep duration, habitual sleep efficiency, sleep disturbances, use of sleeping medication, and daytime dysfunction. A score of $\geq 5$ identifies clinically significant sleep complaints.

The Dutch Sleep Disorders Questionnaire (SDQ, [88] is a questionnaire with 75 questions which can be graded by five categories ranging from 'never' to 'very often or always'. The questionnaire is derived from the Sleep Diagnostic Questionnaire by Douglass et al [89]. The questions cover 6 dimensions of sleep(-related) disorders: insomnia, periodic limb movement syndrome, excessive daytime sleepiness, narcolepsy, psychiatry, and sleep apnea syndrome. For each dimension, a subscale score can be calculated from the relevant items in the questionnaire, ranging from 1 to 5 . For all dimensions the cut-off score of $\geq 3$ indicate a clinically sleep (-related) problem.

'Ervaren Druk door Informele Zorg' (Self-Perceived Pressure from Informal Care, EDIZ) consists of 9 items which form a one-dimensional hierarchical scale, ranging from low to high caregiver burden [90]. Each statement can be answered with 'Yes!' 'Yes' 'More or less' (1 point), 'No', or 'No!' (0 points) creating a total score ranging from 0 to 9 . A score of $\geq 4$ indicates that burden is present. There are no published norm values for this scale.
The Zarit Burden Interview (ZBI) assesses the level of burden experienced by the principal caregiver of patients with dementia [91]. The principal caregiver classifies 12 propositions into four grades, ranging from 'never' to 'very much'. This yields a score with a maximum of 48 points, divided into categories where $0-10$ points imply little or no burden, 11-20 points entails mild to moderate burden, 21-30 points indicate moderate to severe burden and $31-48$ points means severe burden.

\section{List of abbreviations}

AD: Alzheimer Disease; AIS: Athens Insomnia Scale; ADL: Activities of Daily Living; AMP: Absolute Amplitude Measure; BLT: Bright light therapy; DSM-IV: Diagnostic and Statistical Manual of Mental Disorders, 4th Edition; EDIZ: Ervaren Druk door Informele Zorg (SelfPerceived Pressure from Informal Care); GDS: Geriatric Depression Scale; HPA-axis: hypothalamus-pituitary adrenocortical axis; HR: heart rate; IS: Interdaily Stability; IV: Intradaily Variability: L5: 5 hours of lowest activity; M10: 10 hours of highest activity; NINCDSADRDA: National Institute of Neurological and Communicative Disorders and Stroke and the Alzheimer's Disease and Related Disorders Association (now known as the Alzheimer's Association); PSQI: Pittsburg Sleep Quality Inventory; RA: Relative Amplitude; SAD: seasonal affective disorder; SDL: Sleep Diagnose List; SE: Sleep efficiency; SCN: Suprachiasmatic nucleus; TST: total sleep time; ZBT: Zarit Burden Interview.

\section{Acknowledgements}

Funded by the Prevention Program of the Dutch Scientific Organization (ZonMW), The Hague, project 0028.300.30 and the Netherlands Organization of Scientific Research (NWO), The Hague, projects 453-07-001.

\section{Author details}

${ }^{1}$ Netherlands Institute for Neuroscience, an Institute of the Royal Netherlands Academy of Arts and Sciences, Meibergdreef 47, 1105 BA Amsterdam, the Netherlands. ${ }^{2}$ Department of Neurology, VU University Medical Center, De Boelelaan 1117, 1081 HV Amsterdam, the Netherlands. ${ }^{3}$ Department of Integrative Neurophysiology, VU University Amsterdam, De Boelelaan 1085 , 1081 HV Amsterdam, The Netherlands. ${ }^{4}$ Department of Neurology, Leiden University Medical Center, Room T5-32, PO Box 9600, 2300 RC Leiden, The Netherlands.

\section{Authors' contributions}

EM participated in the study design, patient recruitment and trial coordination, and drafted the manuscript.

PS participated in the study design, patient recruitment and trial coordination.

EJWS is the principal investigator and initiator of the study, obtained

funding, designed the study and supervised and participated in writing the manuscript. All authors have read and approved the final manuscript.

\section{Competing interests}

The authors declare that they have no competing interests.

Received: 21 October 2009

Accepted: 23 February 2010 Published: 23 February 2010 
References

1. Lawhorne L: Depression in the older adult. Primary Care 2005, 32(3):777-92.

2. Alexapoulos GS: Depression in the elderly. Lancet 2005, 365(9475):1961-1970.

3. Beekman AT, Copeland JR, Prince MJ: Review of community prevalence of depression in later life. British Journal of Psychiatry 1999, 174:307-311.

4. Brookmeyer R, Johnson E, Ziegler-Graham K, Arrighi HM: Forecasting the global burden of Alzheimer's disease. Alzheimers \& Dementia 2007 3:186-191.

5. Ancoli-Israel S, Klauber MR, Gillin JC, Campbell SS, Hofstetter CR: Sleep in non-institutionalized Alzheimer's disease patients. Aging (Milano) 1994 6(6):451-458

6. Ancoli-Israel S, Klauber MR, Jones DW, Kripke DF, Martin J, Mason W, PatHorenczyk R, Fell R: Variations in circadian rhythms of activity, sleep, and light exposure related to dementia in nursing-home patients. Sleep 1997, 20(1):18-23.

7. Ancoli-Israel S, Clopton P, Klauber MR, Fell R, Mason W: Use of wrist activity for monitoring sleep/wake in demented nursing-home patients. Sleep 1997, 20(1):24-27.

8. Van Someren EJ, Hagebeuk EE, Lijzenga C, Scheltens P, de Rooij SE, Jonker C, Pot AM, Mirmiran M, Swaab DF: Circadian rest-activity rhythm disturbances in Alzheimer's disease. Biological Psychiatry 1996, 40(4):259-270.

9. Witting W, Kwa IH, Eikelenboom P, Mirmiran M, Swaab DF: Alterations in the circadian rest-activity rhythm in aging and Alzheimer's disease. Biological Psychiatry 1990, 27(6):563-572.

10. Bhatt MH, Podder N, Chokroverty S: Sleep and neurodegenerative diseases. Seminars in Neurology 2005, 25(1):39-51.

11. Martin J, Marler M, Shochat T, Ancoli-Israel S: Circadian rhythms of agitation in institutionalized patients with Alzheimer's disease. Chronobiolical International 2000, 17(3):405-418

12. Lyketsos CG, Veiel LL, Baker A, Steele C: A randomized, controlled trial of bright light therapy for agitated behaviors in dementia patients residing in long-term care. International Journal of Geriatric Psychiatry 1999, 14(7):520-525.

13. Flint AJ: Delusions in dementia: a review. The Journal of Neuropsychiatry and Clinical Neurosciences 1991, 3(2):121-130.

14. Forstl $\mathrm{H}$ : Neuropathology of behavioral and psychological symptoms of dementia. International Psychogeriatrics 2000, 12:77-81.

15. Wragg RE, Jeste DV: Overview of depression and psychosis in Alzheimer's disease. American Journal of Psychiatry 1989, 146(5):577-587.

16. Lee HB, Lyketsos CG: Depression in Alzheimer's disease: heterogeneity and related issues. Biological Psychiatry 2003, 54(3):353-362.

17. Purandare N, Burns A, Craig S, Faragher B, Scott K: Depressive symptoms in patients with Alzheimer's disease. International Journal of Geriatric Psychiatry 2001, 16(10):960-964.

18. Steinberg M, Sheppard JM, Tschanz JT, Norton MC, Steffens DC, Breitner JC, Lyketsos CG: The incidence of mental and behavioral disturbances in dementia: the cache county study. The Journal of Neuropsychiatry and Clinical Neurosciences 2003, 15(3):340-345.

19. Burns A, Jacoby $R$, Luthert $P$, Levy R: Cause of death in Alzheimersdisease. Age and Ageing 1990, 19(5):341-344.

20. Olin JT, Schneider LS, Katz IR, Meyers BS, Alexopoulos GS, Breitner JC, Bruce ML, Caine ED, Cummings JL, Devanand DP, Krishnan KR, Lyketsos CG, Lyness JM, Rabins PV, Reynolds CF, Rovner BW, Steffens DC, Tariot PN, Lebowitz BD: Provisional diagnostic criteria for depression of Alzheimer disease. American Journal of Geriatric Psychiatry 2002, 10(2):125-128.

21. Olin JT, Katz IR, Meyers BS, Schneider LS, Lebowitz BD: Provisional diagnostic criteria for depression of Alzheimer disease: rationale and background. American Journal of Geriatric Psychiatry 2002, 10(2):129-141.

22. Turro-Garriga O, Lopez-Pousa S, Vilalta-Franch J, Turon-Estrada A, PericotNierga I, Lozano-Gallego M, Hernandez-Ferrandiz M, Soler-Cors O, Planas-Pujol X, Monserrat-Vila S, Garre-Olmo J: A longitudinal study of apathy in patients with Alzheimer's disease. Revista De Neurologia 2009, 48(1):7-13

23. Verkaik $R$, Nuyen J, Schellevis F, Francke A: The relationship between severity of Alzheimer's disease and prevalence of comorbid depressive symptoms and depression: a systematic review. International Journal of Geriatric Psychiatry 2007, 22(11):1063-1086.

24. Shin IS, Carter M, Masterman D, Fairbanks L, Cummings JL: Neuropsychiatric symptoms and quality of life in Alzheimer disease. American Journal of Geriatric Psychiatry 2005, 13(6):469-474.
25. Gonzalez-Salvador T, Lyketsos CG, Baker A, Hovanec L, Roques C, Brandt J, Steele C: Quality of life in dementia patients in longterm care. International Journal of Geriatric Psychiatry 2000, 15(2):181-189.

26. Gaugler JE, Yu F, Krichbaum K, Wyman JF: Predictors of nursing home admission for persons with dementia. Medical Care 2009, 47(2):191-198.

27. Suh GH, Yeon BK, Shah A, Lee JY: Mortality in Alzheimer's disease: a comparative prospective Korean study in the community and nursing homes. International Journal of Geriatric Psychiatry 2005, 20(1):26-34.

28. Arkin S, Mahendra N: Insight in Alzheimer's patients: results of a longitudinal study using three assessment methods. American Journal of Alzheimer Disease and Other Dementias 2001, 16(4):211-224.

29. Zubenko GS: Neurobiology of major depression in Alzheimer's disease. International Psychogeriatrics 2000, 12:217-230.

30. Zweig RM, Ross CA, Hedreen JC, Steele C, Cardillo JE, Whitehouse PJ, Folstein MF, Price DL: The neuropathology of aminergic nuclei in Alzheimers-disease. Annals of Neurology 1988, 24(2):233-242.

31. Syed A, Chatfield M, Matthews E, Harrison R, Brayne C, Esiri MM: Depression in the elderly: pathological study of raphe and locus ceruleus. Neuropathology and Applied Neurobiology 2005, 31(4):405-413.

32. Hoogendijk WJG, Sommer IEC, Pool CW, Kamphorst W, Hofman MA, Eikelenboom P, Swaab DF: Lack of association between depression and loss of neurons in the locus coeruleus in Alzheimer disease. Archives of General Psychiatry 1999, 56(1):45-51

33. Migliorelli R, Teson A, Sabe L, Petracchi M, Leiguarda R, Starkstein SE: Prevelance and correlates of dysthymia and major depression among patients with Alzheimer-disease. American Journal of Psychiatry 1995 152(1):37-44

34. Cole $M G$, Dendukuri N: Risk factors for depression among elderly community subjects: A systematic review and meta-analysis. American Journal of Psychiatry 2003, 160(6):1147-1156

35. Cho HJ, Lavretsky H, Olmstead R, Levin MJ, Oxman MN, Irwin MR: Sleep Disturbance and Depression Recurrence in Community-Dwelling Older Adults: A Prospective Study. American Journal of Psychiatry 2008, 165(12):1543-1550

36. Roberts RE, Shema SJ, Kaplan GA, Strawbridge WJ: Sleep complaints and depression in an aging cohort: A prospective perspective. American Journal of Psychiatry 2000, 157(1):81-88.

37. Ford DE, Kamerow DB: Epidemiologic-study of sleep disturbances and psychiatric-disordes - an opprtunity for prevention. JAMA 1989, 262(11):1479-1484

38. Ancoli-Israel S, Poceta JS, Stepnowsky C, Martin J, Gehrman P: Identification and treatment of sleep problems in the elderly. Sleep Med Rev 1997 1(1):3-17.

39. Buijs RM, Kalsbeek A: Hypothalamic integration of central and peripheral clocks. Nature Review Neuroscience 2001, 2(7):521-526.

40. Swaab DF, Fliers E, Partiman TS: The suprachiasmatic nucleus of the human brain in relation to sex, age and senile dementia. Brain Research 1985, 342(1):37-44

41. Harper DG, Stopa EG, Kuo-Leblanc V, McKee AC, Asayama K, Volicer L, Kowall N, Satlin A: Dorsomedial SCN neuronal subpopulations subserve different functions in human dementia. Brain 2008, 131:1609-1617.

42. Vitiello MV, Borson S: Sleep disturbances in patients with Alzheimer's disease: epidemiology, pathophysiology and treatment. CNS Drugs 2001 15(10):777-796.

43. Riemersma-van der Lek RF, Swaab DF, Twisk J, Hol EM, Hoogendijk WJ, Van Someren EJ: Effect of bright light and melatonin on cognitive and noncognitive function in elderly residents of group care facilities: a randomized controlled trial. JAMA 2008, 299(22):2642-2655.

44. Ancoli-Israel S, Gehrman P, Martin JL, Shochat T, Marler M, Corey-Bloom J, Levi L: Increased light exposure consolidates sleep and strengthens circadian rhythms in severe Alzheimer's disease patients. Behavioural Sleep Medicine 2003, 1(1):22-36

45. Satlin A, Volicer L, Ross V, Herz L, Campbell S: Bright light treatment of behavioral and sleep disturbances in patients with Alzheimer's disease. American Journal of Psychiatry 1992, 149(8):1028-1032.

46. Dawson P: Bright light treatment for people with Alzheimer's disease. Perspectives 1999, 23(1):25-26.

47. Okumoto $Y$, Koyama E, Matsubara H, Nakano T, Nakamura R: Sleep improvement by light in a demented aged individual. Psychiatry and Clinical Neuroscience 1998, 52(2):194-196. 
48. Dowling GA, Mastick J, Hubbard EM, Luxenberg JS, Burr RL: Effect of timed bright light treatment for rest-activity disruption in institutionalized patients with Alzheimer's disease. International Journal of Geriatric Psychiatry 2005, 20(8):738-743.

49. Colenda CC, Cohen W, McCall WW, Rosenquist PB: Phototherapy for patients with Alzheimer disease with disturbed sleep patterns: Results of a community-based pilot study. Alzheimer Disease \& Associated Disorders 1997, 11(3):175-178.

50. Ohashi Y, Okamoto N, Uchida K, lyo M, Mori N, Morita Y: Daily rhythm of serum melatonin levels and effect of light exposure in patients with dementia of the Alzheimer's type. Biological Psychiatry 1999, 45(12):1646-1652.

51. Dowling GA, Hubbard EM, Mastick J, Luxenberg JS, Burr RL, Van Someren EJ: Effect of morning bright light treatment for rest-activity disruption in institutionalized patients with severe Alzheimer's disease. Int Psychogeriatr 2005, 17(2):221-236.

52. Gasio PF, Krauchi K, Cajochen C, van Someren E, Amrhein I, Pache M, Savaskan E, Wirz-Justice A: Dawn-dusk simulation light therapy of disturbed circadian rest-activity cycles in demented elderly. Experimental Gerontology 2003, 38(1-2):207-216.

53. Dowling GA, Graf CL, Hubbard EM, Luxenberg JS: Light treatment for neuropsychiatric behaviors in Alzheimer's disease. Western Journal of Nursing Research 2007, 29(8):961-975.

54. Terman M, Terman JS: Light therapy for seasonal and nonseasonal depression: Efficacy, protocol, safety, and side effects. Cns Spectrums 2005, 10(8):647.

55. Even C, Schroder CM, Friedman S, Rouillon F: Efficacy of light therapy in nonseasonal depression: A systematic review. Journal of Affective Disorders 2008, 108(1-2):11-23.

56. Levitt AJ, Joffe RT, Moul DE, Lam RW, Teicher MH, Lebegue B, Murray MG, Oren DA, Schwartz P, Buchanan A, et al: Side-effects of light therapy in seasonal affective-disorder. American Journal of Psychiatry 1993, 150(4):650-652.

57. Gallin PF, Terman M, Reme CE, Rafferty B, Terman JS, Burde RM: Ophthalmologic examination of patients with seasonal affective disorder, before and after bright light therapy. American Journal of Ophthalmology 1995, 119(2):202-210.

58. American Psychiatric Association: Diagnostic and Statistical Manual of Mental Disorders. Washington 1994, IV.

59. McKhann G, Drachman D, Folstein M, Katzman R, Price D, Stadlan EM: Clinical diagnosis of Alzheimer's disease: report of the NINCDS-ADRDA Work Group under the auspices of Department of Health and Human Services Task Force on Alzheimer's Disease. Neurology 1984, 34(7):939-944.

60. Petersen RC, Stevens JC, Ganguli M, Tangalos EG, Cummings JL, DeKosky ST: Practice parameter: Early detection of dementia: Mild cognitive impairment (an evidence-based review) - Report of the Quality Standards Subcommittee of the American Academy of Neurology. Neurology 2001, 56(9):1133-1142.

61. Folstein MF, Folstein SE, McHugh PR: "Mini-mental state". A practical method for grading the cognitive state of patients for the clinician. Journal of Psychiatric Research 1975, 12(3):189-198.

62. Twisk J: Applied Longitudinal Data Analysis for Epidemiology. Camebridge: Cambridge University Press 2003.

63. Petkova E, Teresi J: Some statistical issues in the analyses of data from longitudinal studies of elderly chronic care populations. Psychosomatic Medicine 2002, 64(3):531-547.

64. Gibbons RD, Hedeker D, Elkin I, Waternaux C, Kraemer HC, Greenhouse JB, Shea MT, Imber SD, Sotsky SM, Watkins JT: Some Conceptual and Statistical Issues in Analysis of Longitudinal Psychiatric Data Application to the Nimh Treatment of Depression Collaborative Research-Program Dataset. Archives of General Psychiatry 1993, 50(9):739-750.

65. Clements $A D$, Parker $C R$ : The relationship between salivary cortisol concentrations in frozen versus mailed samples. Psychoneuroendocrinology 1998, 23(6):613-616.

66. Chen YM, Cintron NM, Whitson PA: Longt-term storage of salivary cortisol samples at room-temperature. Clinical Chemistry 1992, 38(2):304-304.

67. Garde AH, Hansen AM: Long-term stability of salivary cortisol. Scandinavian Journal of Clinical and Laboratory Investigation 2005, 65(5):433-436.

68. Van Someren EJ, Kessler A, Mirmiran M, Swaab DF: Indirect bright light improves circadian rest-activity rhythm disturbances in demented patients. Biological Psychiatry 1997, 41(9):955-963.
69. Van Someren EJW: Improving actigraphic sleep estimates in insomnia and dementia: how many nights?. Journal of Sleep Research 2007, 16(3):269-275

70. Van Someren EJ: Improved actigraphic sleep estimates by automated lights out and rise time recording and longer assessment duration. Journal of Sleep Research 2006, 15:171-171.

71. Hoekert M, Riemersma-van der Lek RF, Swaab DF, Kaufer D, Van Someren EJW: Comparison between informant-observed and actigraphic assessments of sleep-wake rhythm disturbances in demented residents of homes for the elderly. American Journal of Geriatric Psychiatry 2006, 14(2):104-111

72. Carvalho-Bos SS, Riemersma-van der Lek RF, Waterhouse J, Reilly T, Van Someren EJW: Strong association of the rest-activity rhythm with wellbeing in demented elderly women. American Journal of Geriatric Psychiatry 2007, 15(2):92-100.

73. Van Marken Lichtenbelt WD, Daanen HA, Wouters L, Fronczek R, Raymann RJ, Severens NM, Van Someren EJ: Evaluation of wireless determination of skin temperature using iButtons. Physiology \& Behavior 2006, 88(4-5):489-497.

74. Raymann RJ, Swaab DF, Van Someren EJ: Cutaneous warming promotes sleep onset. American Journal of Physiology - Regulatory, Integrative and Comparative Physiology 2005, 288(6):R1589-1597.

75. Lezak MD, Howieson DB, Loring DW, Hannay HJ, Fischer JS: Neuropsychological Assessment. New York: Oxford University Press, 4 2004.

76. Wechsler D: Weschsler Adult Intelligence Scale-III. San Antonio: The Psychological Corporation 1997.

77. Lindeboom J, Schmand B, Tulner L, Walstra G, Jonker C: Visual association test to detect early dementia of the Alzheimer type. Journal of Neurology Neurosurgery and Psychiatry 2002, 73(2):126-133.

78. Stroop JR: Studies of interference in serial verbal reactions. Journal of Experimental Psychology 1935, 18:643-662.

79. Luteijn F, Ploeg Van Der FAE: Groninger Intelligentie Test (GIT). Lisse, Swets \& Zeitlinger 1983

80. Reitan RM: The Relation of the Trail Making Test to Organic Brain Damage. Journal of Consulting Psychology 1955, 19(5):393-394.

81. Corwin J, Bylsma FW: Psychological-examination of traumatic encephalopathy - The complesx figure copy test. Clinical Neuropsychologist 1993, 7(1):3-21.

82. Overdorp EPL: Validity of the Amsterdamse Dementiescreeningstest. 1989, 1(44):41-44.

83. Wechsler D: Wechsler Adult Intelligence Scale-Revised. San Antonio: The Psychological Corporation 1981

84. Wilson BA, Evans JJ, Alderman N, Burgess PW, Emslie H: Behavioral Assessment Of Dysexecutive Syndrome. Psychology Press 1997.

85. Yesavage JA, Brink TL, Rose TL, Lum O, Huang V, Adey M, Leirer VO: Development and validation of a geriatric depression screening scale - a preliminary-report. Journal of Psychiatric Research 1983, 17(1):37-49.

86. Soldatos CR, Dikeos DG, Paparrigopoulos TJ: Athens Insomnia Scale: validation of an instrument based on ICD-10 criteria. Journal of Psychosomatic Research 2000, 48(6):555-560.

87. Buysse DJ, Reynolds CF, Monk TH, Berman SR, Kupfer DJ: The Pittsburgh Sleep Quality Index - A New Instrument For Psychiatric Practice And Research. Psychiatry Research 1989, 28(2):193-213.

88. Sweere Y, Kerkhof GA, De Weerd AW, Kamphuisen HA, Kemp B, Schimsheimer RJ: The validity of the Dutch Sleep Disorders Questionnaire (SDQ). Journal of Psychosomatic Research 1998 45(6):549-555.

89. Douglass AB, Bornstein R, Nino-Murcia G, Keenan S, Miles L, Zarcone VP Jr, Guilleminault C, Dement WC: The Sleep Disorders Questionnaire. I: Creation and multivariate structure of SDQ. Sleep 1994, 17(2):160-167.

90. Pot AM, van Dyck R, Deeg DJ: [Perceived stress caused by informal caregiving. Construction of a scale]. Tijdschr Gerontol Geriatr 1995, 26(5):214-219.

91. Zarit SH, Reever KE, Bach-Peterson J: Relatives of the impaired elderly: correlates of feelings of burden. Gerontologist 1980, 20(6):649-655.

doi:10.1186/1745-6215-11-19

Cite this article as: Most et al:: Prevention of depression and sleep disturbances in elderly with memory-problems by activation of the biological clock with light - a randomized clinical trial. Trials 2010 11:19. 